\title{
Understanding saving and portfolio choices with predictable changes in assets returns
}

\author{
Christian Gollier ${ }^{1}$ \\ University of Toulouse
}

February 22, 2007

\footnotetext{
${ }^{1}$ I am grateful to André Masson, an anonymous reviewer and seminar participants of the 6th RTN Conference on Ageing held in Frankfurt University for their helpful comments.
} 


\begin{abstract}
We consider an expected-utility-maximizing consumer living two periods who can invest in two assets, one of which is risk free. We do not restrict relative risk aversion to be constant. We first examine the effect that a change in the opportunity set in the second period has on the optimal saving in the first period. We show that an increase in the future risk free rate (keeping the equity premium unchanged) reduces savings if relative risk aversion is uniformly larger than unity. An increase in the equity premium or a reduction in the volatility of the risky asset raises savings if the index of cautiousness, i.e., the derivative of absolute risk tolerance, is smaller than unity. In a second stage, we use these results to determine the sign of the hedging demand for the risky asset for the following three types of predictability: predictable changes in the interest rate, mean-reversion in stock returns, and predictable changes in volatility. Depending upon the type of predictability under scrutiny, what matters to sign the hedging demand is whether relative risk aversion or cautiousness is smaller or larger than unity.
\end{abstract}

Keywords: predictability, precautionary saving, cautiousness, mean reversion, stochastic volatility, strategic asset allocation. 


\section{Introduction}

The investment opportunity set available to investors is not constant over time. Moreover, it fluctuates stochastically, and these fluctuations are serially correlated with economic and financial variables that are observable, i.e., they are predictable. Three types of predictable changes in the investment opportunity set have been pointed out in the literature. First, the short term interest rate is negatively correlated with the past return of long-term bonds. The theory of the term structure of interest rates (Vasicek (1977), Cox, Ingersoll and Ross (1985)) describes this relationship. Second, there is evidence of mean-reversion in stock returns, as first shown by Poterba and Summers (1988). This implies that the future equity premium is negatively correlated with the past returns of equity. Third, French, Schwert and Stambaugh (1987) have shown that the volatility of stock returns is predictable, because it is negatively correlated with past stock returns.

The existence of these various forms of predictability in assets returns has generated a revival of interest in the Merton's notion of intertemporal hedging demand (see Merton $(1969,1971)$ and Mossin (1968)). Campbell and Viceira (2002) provide an overview of this recent literature, and they quantify the importance of the impact of predictability on the optimal portfolio structure. The predictability of future asset returns has two important consequences for the optimal dynamic portfolio strategy. First, a change in the instantaneous interest rate, in the equity premium or in the volatility of asset returns modifies the optimal structure of the portfolio. For example, an increase in the volatility of the stocks returns should typically reduce the demand for stocks by all risk-averse investors. ${ }^{1}$ This "market timing" of the optimal portfolio would be the typical reaction of myopic investors. But, when determining their optimal portfolio today, rational investors should also take into account of the change in the set of future investment opportunities. This yields a second term to the assets demand, which is usually referred to as the "hedging demand".

\footnotetext{
${ }^{1}$ This comparative static property does hold only under some conditions on the investor's utility function, as first shown by Rothschild and Stiglitz (1971). Gollier (1995) characterizes the stochastic dominance order that guarantees that all risk-averse agents reduce their demand for the risky asset. Abel (2002) provides some sufficient conditions for a change in the distribution of returns to reduce the equilibrium expected return of the risky asset.
} 
The objective of this paper is to derive sufficient conditions for signing this hedging demand. There is an important literature on this question. Most papers solve the problem by restricting the set of utility functions to those that exhibit linear risk tolerance. We define the index of cautiousness as the derivative of risk tolerance with respect to consumption. An important special case of utility functions with linear risk tolerance is given by power functions, for which (constant) relative risk aversion equals the inverse of the index of cautiousness. Under this condition, some simple and convergent comparative static properties emerge. In the special case of the logarithmic utility function, i.e., when the index of cautiousness equals unity, the hedging demand for the risky asset is zero. In this case, myopia is always optimal. On the contrary, when the index of cautiousness is constant and smaller than unity, one obtains the intuitive sign for the hedging demand. For example, Brennan, Schwartz and Lagnado (1997) have shown that it implies that the rational investor should invest more in long bonds than the myopic agent to take into account of the negative correlation between the instantaneous (risky) return of this asset and the future interest rate. Under the same assumption on the investor's preferences, Kim and Omberg (1996) have shown that the existence of mean reversion in stock returns yields a positive hedging demand for stocks. In these two illustrations, the idea is that the risky asset is safer in the long run due to the negative serial correlation of returns. When the index of cautiouness is a constant larger than unity, the counterintuitive sign holds for the hedging demand of the risky asset. For example, the existence of mean reversion in stock returns should induce investors with a long horizon to reduce their demand for stocks if their constant relative risk aversion is less than unity.

The main objective of this paper is twofold. First, using a two-period model, we generalize these results by considering any concave utility functions, not only those that exhibit linear risk tolerance, i.e., constant cautiousness. Second, we provide simple intuitions to these results. To do this, we consider the simplest dynamic model with predictability. This model entails only two decision dates and two assets, one of which is risk free. The investor is endowed with some initial wealth which is invested over the two periods to finance consumption at the end of each period. At the beginning of the second period, the agent can rebalance her portfolio. We measure the impact of the predictability of the change of the investment opportunity set in the second period on the optimal portfolio in the first period. To do this, we 
need to characterize the value function of the wealth accumulated from the first period investment. By backward induction, this value function equals the maximum expected utility of wealth, which depends upon the distribution of assets returns available during the second period. Suppose that the stochastic structure of predictability is such that the period-2 distribution of assets returns is "more favorable" when the accumulated wealth is lower. By "more favorable", we mean that it reduces the marginal value of wealth. In that case, the predictability of the second period assets returns reduces the marginal value of wealth where it is large (low wealth), and it raises it where it is small (large wealth). In short, this form of predictability reduces the concavity of the value function, thereby yielding a positive demand for the risky asset in the first period. From this discussion, we see that the relevant question is when does a change in the opportunity set can be considered as favorable, i.e. when does it reduce the marginal value of wealth?

An important message emerges from this work. In spite of the apparent homogeneity of the above-mentioned literature on the sign of the hedging demand, the mechanisms at work are quite different in nature. An increase either in the return of the riskless asset or in the expected return of the risky asset has two contradictory effects on the marginal value of wealth. In the case of an increase in the interest rate, keeping the equity premium unchanged, there is a positive substitution effect and a negative wealth effect, the latter being increasing in the investor's risk aversion. According to this observation, we show that the wealth effect dominates the substitution effect if relative risk aversion is uniformly larger than unity. Consider alternatively the effect of an increase in the expected return of the risky asset on the marginal value of wealth. Compared to the effect of an increase in the interest rate, there is here an additional positive effect due to the rebalancement in favor of the risky asset. An increase in the riskiness of future wealth raises the expected marginal utility if marginal utility is convex, an assumption referred to as "prudence" by Kimball (1990). This positive precautionary effect must be small enough in order to guarantee that an increase in the equity premium reduces the marginal value of wealth. We show that the precise condition for this is that relative prudence be uniformly smaller than twice the relative risk aversion. It happens that for constant relative risk aversion $\gamma$, relative prudence equals $1+\gamma$, which implies that the above condition is equivalent to $\gamma>1$ for this specific set of utility functions. Different types of predictability generally lead to different sufficient conditions to sign the hedging demand. 
These conditions are identical only in the special case of constant relative risk aversion.

Gollier (2004) examined the same problem in a different framework. The main difference is that Gollier (2004) assumes that financial markets are complete, whereas we assume here that there are only two assets. The assumption of the existence of only two aggregate assets, stocks and bonds, makes this paper closer to the existing literature on predictability. Our results are also easier to interpret when there are only two assets. For example, Gollier (2004) is confronted with the difficulty of defining the notion of a riskier price kernel of Arrow-Debreu securities. If our two-asset model is easier to interpret, the proofs of our results are more complex to derive. It has been a surprise to us that the two models lead to the same qualitative conditions on the utility function.

We have explained above why the core of the paper is about deriving the conditions under which a change in the distribution of future returns reduces the marginal value of wealth. This work has its own interest for solving a different dynamic decision problem. Indeed, suppose that the first period problem of the agent is not a portfolio choice as assumed above, but rather a consumption-saving problem similar to the one examined by Drèze and Modigliani (1972) and Sandmo (1970). The agent must determine how much to consume in the first period from initial wealth, the remaining being invested in a risk free asset and in a risky asset. In this alternative decision problem, the question is to determine the relationship between the optimal saving and the distribution of assets returns. We examine this alternative question in the first part of this paper because it is a side result of the characterization of the relationship between the distribution of assets returns in the second period and the marginal value of wealth. Obviously, any change in this distribution that reduces the marginal value of wealth reduces the optimal saving in the first period. Section 2 is devoted to the analysis of the saving problem, whereas section 3 deals with the dynamic saving-portfolio problem. 


\section{The effect of optimism and doubts on sav- ing}

A consumer lives for two periods. She is endowed with wealth $w_{0}>0$ at the beginning of the first period. The agent has no future labour income. From $w_{0}$, the agent saves $s$ and consumes the remaining $w_{0}-s$ in the first period. The felicity function $u_{0}$ associated to the first period consumption is supposed to be continuously differentiable, increasing and concave. There are two assets in the economy. The first asset is risk free and yields a gross return $\rho>0$. The second asset has a random return $\widetilde{r}$ in excess of the risk free rate. From total saving $s$, an amount $\alpha$ is invested in the risky asset and $s-\alpha$ is invested in the risk free asset. It implies that the agent will consume $s \rho+\alpha \widetilde{r}$ in the second period. The felicity function $u$ associated to the second period consumption is three times continuously differentiable, increasing and concave. It satisfies the Inada condition $\lim _{c \rightarrow 0} u^{\prime}(c)=+\infty$. Notice that this specification entails Samuelson's discounted utility model $u=\beta u_{0}$ as a special case. The consumer's objective at the beginning of the first period is to select $s$ and $\alpha$ in order to maximize her lifetime expected utility:

$$
\max _{s, \alpha} u_{0}\left(w_{0}-s\right)+E u(s \rho+\alpha \widetilde{r}) .
$$

In this section, we are interested in determining the effect of a change in the investment opportunity set characterized by $(\rho, \widetilde{r})$ on the optimal saving. Suppose that both the interest rate $\rho$ and the distribution of excess returns $\widetilde{r}$ are parametrized by $\theta$ which is observable by the consumer. It is useful to decompose the above decision problem into two programs. Let us first define the value of saving as the maximum expected utility of future consumption:

$$
v(s, \theta)=\max _{\alpha} E[u(s \rho+\alpha \widetilde{r}) \mid \theta] .
$$

As is well-known, $v$ is increasing and concave in $s$. Using this definition, program (1) can be rewritten as

$$
\max _{s} u_{0}\left(w_{0}-s\right)+v(s, \theta) .
$$

The first-order condition associated to this program is necessary and sufficient:

$$
u_{0}^{\prime}\left(w_{0}-s^{*}\right)=v_{s}\left(s^{*}, \theta\right) .
$$


It yields a unique solution $s^{*}>0$. Using the standard comparative statics method, the optimal saving is increasing in $\theta$ if and only if $v_{s \theta}\left(s^{*}, \theta\right)$ is positive. In short, an increase in $\theta$ raises saving if it raises the marginal value of wealth $v_{s}$. Observe that the envelop theorem implies that the marginal value of wealth equals the product of the gross risk free rate by the expected marginal utility of final consumption:

$$
v_{s}(s, \theta)=\rho E\left[u^{\prime}(s \rho+\alpha(s, \theta) \widetilde{r}) \mid \theta\right] \text {, }
$$

where $\alpha(s, \theta)$ is the optimal demand for the risky asset given $(s, \theta)$ :

$$
E\left[\widetilde{r} u^{\prime}(s \rho+\alpha(s, \theta) \widetilde{r}) \mid \theta\right]=0 .
$$

\subsection{An increase in the future interest rate}

We first examine the effect of an increase in the interest rate $\rho=\theta$ on the optimal saving. The distribution of the excess return of equity is here assumed to be independent of $\theta$. The relationship between the value of saving and the interest rate is given by

$$
v(s, \rho)=\max _{\alpha} E u(s \rho+\alpha \widetilde{r}) .
$$

Let us first consider the classical case without any risky asset, i.e., with $\widetilde{r} \equiv 0$, or equivalently, with $v_{s}(s, \rho)=u^{\prime}(s \rho)$. We obtain in this simple case that $v_{s \rho}\left(s^{*}, \rho\right)=u^{\prime}\left(s^{*} \rho\right)\left[1-R\left(s^{*} \rho\right)\right]$, where $R(z)=-z u^{\prime \prime}(z) / u^{\prime}(z)$ is the ArrowPratt index of relative risk aversion. From this observation, we conclude that when saving must be invested in a risk free asset, an increase in the risk free rate reduces (increases) saving if relative risk aversion is uniformly larger (smaller) than unity. If $w_{0}$ is arbitrary, these are necessary and sufficient conditions. A change in the interest rate $\rho$ yields both a wealth effect and a substitution effect. An increase in $\rho$ reduces the relative price of future consumption, which induces the agent to substitute current consumption by future consumption. In addition, for a given $s$, an increase in $\rho$ raises future consumption. Because the agent has a preference for consumption smoothing over time, this wealth effect induces him to rebalance consumption in favor of current consumption. The strength of this wealth effect is measured by the elasticity of marginal utility, which is given by $R$. This explains why the 
wealth effect dominates the substitution effect $\left(s_{\rho}^{*}<0\right)$ only if relative risk aversion is large enough.

We now consider the more general case with two assets. An increase in the interest rate is likely to induce a rebalancement of the portfolio structure of the consumer. Notice however that, because the distribution of the excess return is unaffected by this change, this rebalancement is only due to the increase in wealth. In particular, an increase in $\rho$ would have no effect on the demand for the risky asset if absolute risk aversion would be constant. In the other cases, the portfolio rebalancement affects the expected marginal utility of future consumption, i.e., it affects the marginal value of wealth or the willingness to save. Using the first-order condition (6), the impact of the increase in $\rho$ on the demand for the risky asset equals

$$
\alpha_{\rho}(s, \rho)=-s \frac{E\left[\widetilde{r} u^{\prime \prime}(s \rho+\alpha \widetilde{r})\right]}{E\left[\widetilde{r}^{2} u^{\prime \prime}(s \rho+\alpha \widetilde{r})\right]} .
$$

It is well known that, if $E \widetilde{r}>0$, the demand for the risky asset is increasing in $s \rho$ if and only if $u$ exhibits decreasing absolute risk aversion. Combining equality (8) with the full differentiation of condition (5) with respect to $\rho$ yields

$$
v_{s \rho}\left(s^{*}, \rho\right)=E u^{\prime}\left(s^{*} \rho+\alpha \widetilde{r}\right)+s^{*} \rho E u^{\prime \prime}\left(s^{*} \rho+\alpha \widetilde{r}\right)-s^{*} \rho \frac{\left[E\left[\widetilde{r} u^{\prime \prime}\left(s^{*} \rho+\alpha \widetilde{r}\right)\right]\right]^{2}}{E\left[\widetilde{r}^{2} u^{\prime \prime}\left(s^{*} \rho+\alpha \widetilde{r}\right)\right]},
$$

where $\alpha=\alpha\left(s^{*}, \rho\right)$. The first term $E u^{\prime}$ in the right-hand side of equation (9) is the substitution effect, which is positive. The second term $s^{*} \rho E u^{\prime \prime}$ is the negative wealth effect. As explained earlier, in the absence of the risky asset $(\widetilde{r} \equiv 0)$, the sign of the sum of these two effects depends upon whether relative risk aversion is smaller or larger than unity. The last term in (9) comes from the ability of the consumer to rebalance her portfolio as a reaction to the change in the interest rate. This rebalancement effect unambiguously raises the willingness to save.

We would like to determine the condition under which $v_{s \rho}$ is negative independent of the distribution of excess returns. This problem can be simplified by the following change of notation: $y=s^{*} \rho, \widetilde{x}=\alpha \widetilde{r}$, and $\widehat{E} f(\widetilde{x})=E f(\widetilde{x}) u^{\prime \prime}(y+\widetilde{x}) / E u^{\prime \prime}(y+\widetilde{x})$. Variables $y$ and $x$ denote respectively the future value of the riskfree portfolio and the excess value of the optimal portfolio, whereas $\widehat{E}$ is some sort of risk-neutral expectation operator. With 
this notation, the marginal value of wealth is reduced by an increase in the risk-free rate if

$$
\widehat{E} \widetilde{x} T(y+\widetilde{x})=0 \Longrightarrow \frac{\widehat{E} T(y+\widetilde{x})}{y} \leq 1-\frac{[\widehat{E} \widetilde{x}]^{2}}{\widehat{E} \widetilde{x}^{2}},
$$

where $T(z)=-u^{\prime}(z) / u^{\prime \prime}(z)$ is the index of absolute risk tolerance evaluated at $z$. Indeed, the equality in (10) is just a rewriting of the first-order condition (6) on $\alpha$, evaluated at $s=s^{*}$. The inequality in (10) corresponds to $v_{s \rho}\left(s^{*}, \rho\right) \leq 0$. We use this characterization in the Appendix to prove the following Proposition. ${ }^{2}$

Proposition 1 An increase in the risk free rate reduces (raises) the marginal value of wealth if relative risk aversion is uniformly larger (smaller) than unity: $v_{s \rho}\left(s^{*}, \rho\right) \leq(\geq) 0$ if $R(c) \geq(\leq) 1$ for all $c \in \mathbb{R}_{+}$.

We conclude that an increase in the interest rate reduces saving if relative risk aversion is larger than unity, and that it raises saving when relative risk aversion is less than unity. This result generalizes what has long been known when the investment opportunity set is limited to a risk free asset.

\subsection{An increase in the equity premium: Optimism}

We now examine the relationship between savings and the equity premium. Let $\widetilde{r}$ be the sum of the equity premium $\theta$ and a zero-mean noise $\widetilde{q}$. When the equity premium $\theta$ is zero, it is optimal not to invest in the risky asset. When the equity premium is positive, the demand for the risky asset becomes positive. How does this increase in the equity premium affect the willingness to save? We can identify two contradictory effects. The first one is a wealth effect: the investment in the risky asset with a positive risk premium raises the expected future consumption. Because of the willingness to smooth consumption over time, this wealth effect tends to reduce savings in the first period. The second effect comes from the optimal rebalancement of the asset portfolio in favor of the risky asset. The uncertainty on the portfolio return affects the willingness to save. Relying on the precautionary saving motive,

\footnotetext{
${ }^{2}$ Bhamra and Uppal (2006) obtained a similar result in a recursive utility framework with power functions.
} 
the intuition suggests that taking into account of this uncertainty has a positive effect on the willingness to save. This induced portfolio rebalancement yields a precautionary effect. Notice that there is no substitution effect, since the relative price of future sure consumption is unchanged.

The portfolio problem is written as

$$
v(s, \theta)=\max _{\alpha} E u(s \rho+\alpha(\widetilde{q}+\theta)) .
$$

We hereafter suppose that $E \widetilde{q}+\theta$ is positive, so that the optimal $\alpha$ is positive. Fully differentiating the first-order condition (6) implies that

$$
\alpha_{\theta}=-\frac{E u^{\prime}(s \rho+\alpha(\widetilde{q}+\theta))+\alpha E\left[(\widetilde{q}+\theta) u^{\prime \prime}(s \rho+\alpha(\widetilde{q}+\theta))\right]}{E\left[(\widetilde{q}+\theta)^{2} u^{\prime \prime}(s \rho+\alpha(\widetilde{q}+\theta))\right]} .
$$

It can easily be proved that $\alpha_{\theta}$ is positive under DARA. As it is intuitive, the demand for the risky asset is increasing with the expected excess return. Fully differentiating $v_{s}(s, \theta)$ given by $(5)$ with respect to $\theta$ yields

$$
\rho^{-1} v_{s \theta}(s, \theta)=\alpha E u^{\prime \prime}(s \rho+\alpha(\widetilde{q}+\theta))+\alpha_{\theta} E\left[(\widetilde{q}+\theta) u^{\prime \prime}(s \rho+\alpha(\widetilde{q}+\theta))\right],
$$

We recognize in the right-hand side the wealth effect and the rebalancement/precautionary effect. Replacing $\alpha_{\theta}$ in the above equation by its expression given by (12), we obtain that

$$
\alpha^{-1} \rho^{-1} v_{s \theta}(s, \theta)=E u^{\prime \prime}(y+\widetilde{x})-\frac{E u^{\prime}(y+\widetilde{x}) E \widetilde{x} u^{\prime \prime}(y+\widetilde{x})}{E \widetilde{x}^{2} u^{\prime \prime}(y+\widetilde{x})}-\frac{\left[E \widetilde{x} u^{\prime \prime}(y+\widetilde{x})\right]^{2}}{E \widetilde{x}^{2} u^{\prime \prime}(y+\widetilde{x})},
$$

where $y=s \rho$ and $\widetilde{x}=\alpha(\widetilde{q}+\theta)$. Using the same risk-neutral expectation operator $\widehat{E} f(\widetilde{x})=E f(\widetilde{x}) u^{\prime \prime}(y+\widetilde{x}) / E u^{\prime \prime}(y+\widetilde{x})$ as defined in the previous section, the marginal value of wealth is decreasing with the expected excess return $\theta$ if and only if the following condition is satisfied:

$$
\widehat{E} \widetilde{x} T(y+\widetilde{x})=0 \Longrightarrow \widehat{E} \widetilde{x} \widehat{E} T(y+\widetilde{x}) \geq[\widehat{E} \widetilde{x}]^{2}-\widehat{E} \widetilde{x}^{2}
$$

The following proposition exhibits the condition under which this property holds. It relies on the index of cautiousness which is defined by $C(z)=$ $T^{\prime}(z)$, or equivalently, $C(z)=\left[u^{\prime}(z) u^{\prime \prime \prime}(z)-u^{\prime \prime 2}(z)\right] / u^{\prime \prime 2}(z)$. The concept of cautiousness has first been introduced by Wilson (1968). It has been used by Leland (1968) and Huang (2004) who showed that investors with a larger index of cautiousness are more willing to purchase portfolio insurance. 
Proposition 2 Define the index of cautiousness as the derivative of the index of absolute risk tolerance. An increase in the equity premium reduces (raises) the marginal value of wealth if the index of cautiousness is uniformly smaller (larger) than unity.

Proof: Suppose that $T^{\prime}$ is uniformly smaller than unity. It implies that $T(y+x)-x$ is decreasing in $x$. By the covariance rule, it implies in turn that

$$
\widehat{E}[\widetilde{x}(T(y+\widetilde{x})-\widetilde{x})] \leq \widehat{E} \widetilde{x} \quad[\widehat{E} T(y+\widetilde{x})-\widehat{E} \widetilde{x}],
$$

or, equivalently, that

$$
-\widehat{E} \widetilde{x}^{2} \leq \widehat{E} \widetilde{x} \widehat{E} T(y+\widetilde{x})-[\widehat{E} \widetilde{x}]^{2}
$$

because $\widehat{E} \widetilde{x} T(y+\widetilde{x})=0$. This implies that $v_{s \theta}$ is negative. The same method can be applied to prove the other case.

A direct consequence of this proposition is that an increase in the expected return of the risky asset reduces savings when cautiousness is less than unity. It may be useful to rewrite the index of cautiousness as

$$
C(z)=P(z) T(z)-1,
$$

where $P(z)=-u^{\prime \prime \prime}(z) / u^{\prime \prime}(z)$ is the index of absolute prudence defined by Kimball (1990) to measure the intensity of the precautionary saving motive. It is an index of convexity of marginal utility, which measures the impact of a zero-mean risk on the marginal value of wealth, i.e. the expected marginal utility of future consumption. Observe that $C$ is less than unity if and only if absolute prudence is less than twice the absolute risk aversion $1 / T$. From this observation, we can derive an intuition to Proposition 2. The increase in the equity premium has a negative wealth effect on initial savings. The intensity of this wealth effect is measured by the willingness to smooth consumption over time given by absolute risk aversion $1 / T$. Because the increase in the equity premium induces the agent to take more risk, there is also a positive precautionary effect on savings that is measured by $P$, as shown by Kimball (1990). Requiring that $P$ be smaller than $2 / T$ has the intuitive meaning that the precautionary effect be small enough compared to the wealth effect.

Both propositions 1 and 2 provide a condition for an unambiguous reduction of the marginal value of wealth due to the increase in the expected 
return of one of the two assets. When the return of the risk free asset is increased, the condition is that relative risk aversion be smaller than unity. When the expected return of the risky asset is increased, the condition is that the index of cautiousness be smaller than unity. These two conditions have a different nature. In particular, the second one refers to the third derivative of the utility function, whereas the first one is limited to risk aversion. It is easy to find utility functions that satisfy the first condition and not the other, and vice versa. However, there exists a family of functions for which the two conditions are equivalent. Suppose that relative risk aversion $R(z)=\gamma$ is constant, i.e. $u(z)=z^{1-\gamma} /(1-\gamma)$. For that family of functions, the index of cautiousness $C(z)$ equals $\gamma^{-1}$. It implies that when relative risk aversion is constant, the index of cautiousness equals the inverse of relative risk aversion. This yields the following result.

Corollary 1 Suppose that relative risk aversion is constant. If relative risk aversion is larger (less) than unity, the marginal value of wealth is reduced (increased) by an increase in the risk free rate and by an increase in the equity premium.

Observe that $R(z) \geq 1$ is equivalent to $T(z) \leq z$, whereas $C(z) \leq 1$ is equivalent to $T^{\prime}(z) \leq 1$. If we assume that absolute risk aversion tends to infinity when wealth tends to zero, i.e., that $T(0)=0$, then, obviously the second condition implies the first one. In this sense, condition $C \leq 1$ is stronger than condition $R \geq 1$. This observation leads to the following result.

Corollary 2 Suppose that absolute risk aversion tends to infinity when wealth tends to zero. Suppose also that an increase in the equity premium always reduces the marginal value of wealth, for any distribution of the excess return and any initial wealth. Then, an increase in the risk free rate always reduces the marginal value of wealth.

The opposite result is not true, except in the special case of constant relative risk aversion.

\subsection{An increase in the volatility of equity returns: Doubts}

We finally consider the effect of an increase in the volatility of equity returns on savings. Abel (2002) refers to this (subjective) increase in volatility as an 
increase in doubts. Suppose that the equity premium $\widetilde{r}$ has a positive mean $\mu$ plus a zero-mean noise $\theta \widetilde{q}$. We are interested by the effect of an increase in $\theta$ on savings in the first period. The value of wealth $v$ is rewritten in this case as

$$
v(z, \theta)=\max _{\alpha} E u(z R+\alpha(\theta \widetilde{q}+\mu)) .
$$

Notice that this problem can be rewritten as

$$
v(z, \theta)=\max _{\alpha} \operatorname{Eu}\left(z R+\alpha\left(\widetilde{q}+\theta^{-1} \mu\right)\right) .
$$

It implies that an increase in volatility $\theta$ has an effect on the marginal value of wealth and saving that is equivalent to a reduction in the equity premium. We thus immediately obtain the following result as a corollary of Proposition 2 .

Proposition 3 The marginal value of wealth is increasing (decreasing) in the volatility of equity returns if the index of cautiousness is uniformly smaller (larger) than unity.

In the standard case of constant relative risk aversion larger than unity, this proposition implies that the willingness to save is increasing in the riskiness of equity returns. When constant relative risk aversion is less than unity, the opposite comparative static property holds.

\section{Predictability of future asset returns and the hedging demand for the risky asset}

In this section, we consider an alternative dynamic choice problem. At the beginning of the first period, the agent is endowed with initial wealth $w_{0}$. She can invest this wealth in a riskfree asset and a risky asset. During period $t, t=1$ or 2 , the riskfree asset has a gross return $\rho_{t}$, whereas the risky asset has a return $\widetilde{r}_{t}$. We assume that the expectation of $\widetilde{r}_{t}$ is positive. At the beginning of the first period, $\rho_{1}$ is certain, whereas the level of $\rho_{2}$ and the distribution of $\widetilde{r}_{2}$ may depend upon the realization of the first period state given by $r_{1}$. This statistical dependency describes the nature of predictability of asset returns. We are interested in determining how does the predictability of future asset returns affect the initial optimal portfolio allocation. 
Within each period $t$, the investor first selects her investment $\alpha_{t}$ in the risky asset. She then determines her consumption level $c_{t}$ after the state of nature $\widetilde{r}_{t}$ is realized. We use backward induction to solve this dynamic portfolio choice problem. Consider the interim date in the first period where $\widetilde{r}_{1}$ is realized, but before consumption takes place. Given the accumulated wealth $w=w_{0} \rho_{1}+\alpha_{1} r_{1}$ at that interim date, and given the state of nature $r_{1}$ realized in the first period, the consumer selects the $\left(c_{1}, \alpha_{2}\right)$ that maximizes the expected utility of her final wealth: ${ }^{3}$

$$
V\left(w, r_{1}\right)=\max _{c_{1}} u_{0}\left(c_{1}\right)+v\left(w-c_{1}, r_{1}\right)
$$

with

$$
v\left(s, r_{1}\right)=\max _{\alpha} E\left[u\left(s \rho_{2}+\alpha \widetilde{r}_{2}\right) \mid r_{1}\right] .
$$

The first-period portfolio problem can then be written as

$$
\alpha_{1}^{*} \in \arg \max _{\alpha_{1}} \quad M\left(\alpha_{1}\right)=E V\left(w_{0} \rho_{1}+\alpha_{1} \widetilde{r}_{1}, \widetilde{r}_{1}\right) .
$$

Because $V$ is concave in its first argument, this program is well-behaved, and its first-order condition is necessary and sufficient. We compare the optimal risky investment $\alpha_{1}^{*}$ in this predictable economy to the optimal risky investment in another economy where future returns are unpredictable. In this unpredictable economy, the distribution of returns of the two assets in the first period are as in the predictable economy. But the distribution of returns of the two assets in the second period are unpredictable in the sense that they are independent of $r_{1}$. They are assumed to be those prevailing in the predictable economy conditional to $r_{1}=0$. It implies that the first period portfolio problem in the unpredictable economy can be written as:

$$
\widehat{\alpha}_{1} \in \arg \max _{\alpha_{1}} E V\left(w_{0} \rho_{1}+\alpha_{1} \widetilde{r}_{1}, 0\right)
$$

We obtain the following Lemma. ${ }^{4}$

\footnotetext{
${ }^{3}$ In an earlier version of this paper, we considered the dynamic investment problem in which $c_{1}$ is constrained to be zero. The same results hold.

${ }^{4} \mathrm{~A}$ weaker condition to yield the same result is that $v_{s}$ satisfies the following singlecrossing condition $r_{1}\left[v_{s}\left(s, r_{1}\right)-v_{s}(s, 0)\right] \geq 0$ for all $r_{1}$ and all $s$.
} 
Lemma 1 The predictability of future asset returns has a positive (negative) effect on the initial demand for the risky asset if the marginal value of wealth $v_{s}\left(s, r_{1}\right)$ is increasing (decreasing) in $r_{1}$.

Proof: Fully differentiating the first-order condition $u_{0}^{\prime}\left(c_{1}\right)=v_{s}\left(w-c_{1}, r_{1}\right)$ with respect to $r_{1}$ yields

$$
\frac{d c_{1}}{d r_{1}}=\frac{v_{s r_{1}}\left(w-c_{1}, r_{1}\right)}{u_{0}^{\prime \prime}\left(c_{1}\right)+v_{s s}\left(w-c_{1}, r_{1}\right)} .
$$

Fully differentiating in turn the equation $V_{w}\left(w, r_{1}\right)=u_{0}^{\prime}\left(c_{1}\right)$ yields

$$
V_{w r_{1}}\left(w, r_{1}\right)=u_{0}^{\prime \prime}\left(c_{1}\right) \frac{d c_{1}}{d r_{1}}=\frac{u_{0}^{\prime \prime}\left(c_{1}\right)}{u_{0}^{\prime \prime}\left(c_{1}\right)+v_{s s}\left(w-c_{1}, r_{1}\right)} v_{s r_{1}}\left(w-c_{1}, r_{1}\right) .
$$

Because the ratio in the right-hand side of this equality is positive, $V_{w r_{1}}$ and $v_{s r_{1}}$ have the same sign. Suppose that $v_{s}\left(s, r_{1}\right)$ is increasing in $r_{1}$. It implies that $V_{w}$ is increasing in $r_{1}$.

Observe that because $E \widetilde{r}_{1}$ is positive, $\widehat{\alpha}_{1}$ is positive. Because $V$ is concave in its first argument, function $M$ is concave in $\alpha$. It implies that $\alpha_{1}^{*}$ is larger than $\widehat{\alpha}_{1}$ if $M^{\prime}\left(\widehat{\alpha}_{1}\right)$ is positive. Because $V_{w}\left(w, r_{1}\right)$ is increasing in $r_{1}$, we have that

$$
r_{1} V_{w}\left(w_{0} \rho_{1}+\widehat{\alpha}_{1} r_{1}, r_{1}\right) \geq r_{1} V_{w}\left(w_{0} \rho_{1}+\widehat{\alpha}_{1} r_{1}, 0\right)
$$

for all $r_{1}$. It implies that

$$
M^{\prime}\left(\widehat{\alpha}_{1}\right)=E \widetilde{r}_{1} V_{w}\left(w_{0} \rho_{1}+\widehat{\alpha}_{1} \widetilde{r}_{1}, \widetilde{r}_{1}\right) \geq E \widetilde{r}_{1} V_{w}\left(w_{0} \rho_{1}+\widehat{\alpha}_{1} \widetilde{r}_{1}, 0\right)=0 .
$$

The last equality is the first-order condition for $\widehat{\alpha}_{1}$. We concludes that $\alpha_{1}^{*}$ is larger than $\widehat{\alpha}_{1}$. A symmetric proof holds for the case where $v_{s}\left(s, r_{1}\right)$ is decreasing in $r_{1}$

When the marginal value of wealth is increasing in the first-period return of the risky asset, predictability affects the initial attitude towards portfolio risk in a manner similar to a reduction in risk aversion. Indeed, it raises the marginal value of wealth where it is small (large $r_{1}$ ), and it reduces it where it is large (small $r_{1}$ ). This is a situation where the bad luck in the first period is (partially) compensated by a change in the future opportunity set that reduces the marginal value of wealth. In such circumstances, the predictability has a stabilizing effect on $v_{s}$ and $V_{w}$. This has a positive effect on the portfolio risk tolerance in the first period. 
Lemma 1 builds a bridge between the predictability problem under scrutiny in this section and what we have done earlier in this paper. In the next subsections, we combine Lemma 1 with our earlier propositions that characterize the conditions under which a change in the opportunity set increases the marginal value of wealth.

\subsection{Predictable future interest rates}

In this section, we assume that there is no serial correlation in the returns of the risky asset. On the contrary, we assume that the riskfree rate in the second period is unknown at the beginning of the first period. It will be learned during the first period. Finally, we assume that the return of the risky asset in the first period is informative for the return of the riskfree asset in the second period. This situation arises on the market for bonds with different maturities. When considering the first period in isolation, the riskfree asset is the bond with maturity at the end of the first period, whereas the bond with a two-period maturity is risky. Its first period return depends upon the information obtained during the first period about the riskfree rate in the second period. All models of bond pricing predicts that an increase in the expected future interest rate reduces the instantaneous return of long bonds. Thus, there is a negative relationship between $\widetilde{r}_{1}$ and $\rho_{2}$. We compare the optimal portfolio of short and long bonds of two agents with the same degree of risk aversion and the same initial wealth. The first agent is fully rational and takes into account of the negative serial correlation of the long bond. The second agent is myopic in the sense that she believes that the returns of the long bond are independent over time.

The optimal investment in the short and long bonds must take into account of the difference in current returns, but also of the recognition that long bonds have larger short-term returns when future short-term rates are expected to be lower. Thus, long bonds can hedge against changes in the future investment opportunity set. The hedging demand for long bonds is measured by the additional demand from a rational investor in comparison to what would decide a myopic investor treating the long bond as simply another risky asset whose returns would have no serial correlation. Brennan, Schwartz and Lagnado (1997) solve this problem by using a power utility function, together with assuming the pure expectations hypothesis for bond prices. The following proposition provides a more general result. It is more 
general because we do not assume constant relative risk aversion, and because we do not make any restriction on bond pricing other than the assumption that a larger future interest rate reduces the current return of long bonds.

Proposition 4 Suppose that there is a negative relationship between the return of the risky asset in the first period and the riskfree rate in the second period. This form of predictability has a positive (negative) effect on the demand for the risky asset in the first period if relative risk aversion is uniformly larger (smaller) than unity.

Proof: Suppose that relative risk aversion is uniformly larger than unity. We know from Proposition 1 that an increase in $\rho_{2}$ reduces the marginal value of wealth in that case. Because there is a negative relationship between $\rho_{2}$ and $r_{1}$, we obtain that $v_{s}$ is increasing in $r_{1}$. Using Lemma 1 concludes the proof.

Because of the negative correlation between the current and the future return of long-term bonds, the intuition is that investors should hedge the future risk by raising their demand for the long bond in the first period. The above proposition confirms this intuition only when relative risk aversion is larger than unity. Brennan, Schwartz and Lagnado (1997) solve numerically a simple version of this model to motivate their paper. They show that the intuition is correct only if the constant relative risk aversion is larger than unity. Campbell and Viceira (2001) calibrated a more complex version of this model to quantify the optimal mix of short-term and long-term bonds.

When relative risk aversion is smaller than unity, the optimal investment in the long bond is less than the myopic one, i.e., the hedging demand for long bonds is negative. The intuition in that case is that the substitution effect of an increase of the future interest rate dominates the wealth effect, which is proportional to relative risk aversion. This means that consumers are willing to transfer wealth from the states where $\rho_{2}$ is small to those where $\rho_{2}$ is large. The problem is that holding long bonds do exactly the opposite, because long bonds have a smaller return when the future interest rate is larger. This explain the negative demand for long bonds when relative risk aversion is less than unity. 


\subsection{Predictable equity premium}

Suppose alternatively that the interest rate evolves over time in a deterministic way, but that the equity premium in the second period is contingent to the equity return in the first period. The most obvious illustration of this phenomenon is the existence of mean-reversion in equity returns, as documented for example by Poterba and Summers (1988), Campbell (1996), Campbell, Lo and MacKinlay (1997) and Cochrane (2001). Barberis (2000) for example estimates significant predictability of US stocks returns. The implied standard deviation of ten-year returns is 23.7 percents, much smaller than the 45.2 percents value implied by the standard deviation of monthly returns.

Our comparative statics exercise aims at determining the sign of the hedging demand for equity, i.e., the sign of the difference between the dynamically optimal demand for equity and the demand for equity by an investor believing that there is no mean reversion. Because mean-reversion implies that stocks are safer in the long run, the intuition suggests that a rational agent should purchase more stocks in the first period than a myopic agent: $\alpha_{1}^{*} \geq \widehat{\alpha}_{1}$. Campbell and Viceira (1999) and Barberis (2000) numerically estimated this hedging demand. The effect of return predictability on the optimal structure of the initial portfolio is surprising large. For an agent with a relative risk aversion equaling 10 and a ten-year time horizon, the optimal investment in stocks is about $40 \%$ of current wealth without predictability. It goes up to $100 \%$ when mean-reversion is taken into account. These results are obtained either by numerical simulations, or analytically in the special case of constant relative risk aversion. In the next proposition, we show that what really matters is not whether relative risk aversion is large enough, but rather whether the degree of cautiousness is uniformly smaller than unity. This result will allow us to provide an intuition to this literature on mean-reverting asset returns. It generalizes the result obtained by Kim and Omberg (1996) in the special case of linear risk tolerance.

Proposition 5 Suppose that there is a negative relationship between the return of the risky asset in the first period and the equity premium in the second period. This form of predictability has a positive (negative) effect on the demand for the risky asset in the first period if the index of cautiousness is uniformly smaller (larger) than unity.

Proof: Suppose that the index of cautiousness is uniformly smaller than 
unity. We know from Proposition 2 that an increase in the equity premium in the second period reduces the marginal value of wealth in that case. Because there is a negative relationship between the equity premium in the second period and $r_{1}$, we obtain that $v_{s}$ is increasing in $r_{1}$. Using Lemma 1 concludes the proof.

Proposition 5 tells us that what matters for the sign of the hedging demand is whether the index of cautiousness is larger or smaller than unity, or whether risk aversion is sufficiently stronger than prudence. This proposition also provides an intuition for the result: an increase in the equity premium reduces the marginal value of wealth only if the wealth effect is sufficiently stronger than the precautionary effect. Thus, when the first period return is low, i.e., when the marginal value of wealth is large, the increased anticipated equity premium in the second period reduces this marginal value of wealth. This reduces the aversion towards the first period risk. When the precautionary effect dominates the wealth effect, mean reversion raises the marginal value of wealth in the bad first period states, thereby raising risk aversion.

\subsection{Predictable volatility}

Finally, we consider the case of predictable volatility. There is ample evidence that large negative returns tend to be associated with increases in volatility over long periods of time (French, Schwert and Stambaugh (1987)). Chacko and Viceira (2005) shows that when changes in volatility are negatively correlated with excess stock returns, there is a negative hedging demand when constant relative risk aversion is larger than unity. The opposite result holds when constant relative risk aversion is less than unity. In our next proposition, we extend this result to the case of non-constant relative risk aversion, and we show that the relevant condition is whether the index of cautiousness is smaller or larger than unity.

Proposition 6 Suppose that there is a negative relationship between the return of the risky asset in the first period and the volatility of its return in the second period. This form of predictability has a negative (positive) effect on the demand for the risky asset in the first period if the index of cautiousness is uniformly smaller (larger) than unity. 
Proof: Suppose that the index of cautiousness is uniformly smaller than unity. We know from Proposition 3 that an increase in volatility in the second period increases the marginal value of wealth in that case. Because there is a negative relationship between the volatility in the second period and $r_{1}$, we obtain that $v_{s}$ is decreasing in $r_{1}$. Using Lemma 1 concludes the proof.

The increase in volatility has an impact on the marginal value of wealth that is equivalent to a reduction in the equity premium. The relationship between our last two propositions is thus obvious. The same comments apply for these two results.

\section{Conclusion}

When relative risk aversion is uniformly larger than unity, an increase in the future interest rate reduces the marginal value of wealth. This is because the wealth effect - which is measured by the index of relative risk aversion dominates the substitution effect in that case. We conclude from this property that an increase in the interest rate should reduce savings for agent with no labour income. Moreover, given the negative correlation between the instantaneous return of long bonds and the future interest rate, the hedging demand for the long bond is positive. The opposite comparative static results hold when relative risk aversion is uniformly less than unity.

Following Wilson (1968), we defined the index of cautiousness as the derivative of the index of absolute risk tolerance. We showed that when the index of cautiousness is uniformly smaller than unity, an increase in the equity premium also reduces the marginal value of wealth. This is because the wealth effect dominates a precautionary effect in that case. This precautionary effect comes from the fact that an increase in the equity premium induces the agent to raise her risk exposure, which raises the expected marginal utility of consumption if marginal utility is convex, i.e., if the agent is prudent. This observation implies that, when cautiousness is smaller than unity, an increase in the future equity premium reduces initial savings. If there is mean reversion in stock returns, this also implies that there is a positive hedging demand for stocks. The opposite comparative static results hold when the index of cautiousness is uniformly larger than unity.

When relative risk aversion is assumed to be constant, as in most models in dynamic finance, the index of cautiousness is uniformly smaller than unity 
if and only if relative risk aversion is larger than unity. In that specific case, as is well known, signing the hedging demand due to any type of predictability of future assets returns just requires knowing whether relative risk aversion is smaller or larger than unity. We have shown that relaxing the assumption that relative risk aversion is constant allows us to better understand the different intuitions of the above-mentioned results. 


\section{References}

Abel, A.B., (2002), An exploration of the effects of optimism and doubt on asset returns, Journal of Economic Dynamics and Control, 26, 1075-1092.

Barberis, N., (2000), Investing for the long run when returns are predictable, Journal of Finance, 55, 225-64.

Bhamra, H.S. and R. Uppal, (2006), The role of risk aversion and intertemporal substitution in dynamic consumption-portfolio choice with recursive utility, Journal of Economic Dynamic and Control, 30 (6), 967-991.

Brennan, M.J., E.S. Schwartz and R. Lagnado, (1997), Strategic asset allocation, Journal of Economic Dynamics and Control, 21, 1377-1403.

Campbell, J.Y., (1996), Understanding risk and return, Journal of Political Economy, 104, 298-345.

Campbell, J.Y., A.W. Lo and A. C. MacKinlay, (1997), The Econometrics of Financial Markets, Princeton University Press, Princeton.

Campbell, J.Y., and L. Viceira, (1999), Consumption and portfolio decisions when expected returns are time varying, Quarterly Journal of Economics, 114, 433-95.

Campbell, J.Y., and L. Viceira, (2001), Who should buy longterm bonds?, American Economic Review, 91, 99-127.

Campbell, J.Y., and L.M. Viceira, (2002) Strategic asset allocation, Oxford University Press, Oxford.

Chacko, G., and L.M. Viceira, (2005), Dynamic consumption and portfolio choice with stochastic volatility in incomplete markets, Review of Financial Studies, 18 (4).

Cochrane, J.H., (2001), Asset pricing, Princeton University Press, Princeton.

Cox, J., Ingersoll, J., and S. Ross, (1985), A theory of the term structure of interest rates, Econometrica, 53, 385-403. 
Drèze, J.H. and F. Modigliani, (1972), Consumption decisions under uncertainty, Journal of Economic Theory, 5, 308-335.

French, K.R., G.W. Schwert, and R.F. Stambaugh, (1987), Expected stock returns and volatility, Journal of Financial Economics, 19, 3-29.

Gollier, C., (1995), The Comparative Statics of Changes in Risk Revisited, Journal of Economic Theory, 66, 522-536.

Gollier, C., (2004), Optimal Portfolio Risk with First-Order and Second -Order Predictability, Contributions to Theoretical Economics, 4 (1), Article 4, http://www.bepress.com/bejte/contributions/vol4/iss1/art4.

Huang, J. X., (2004), Cautiousness and Tendency to Buy Options, Lancaster University Management School Working Paper No. 2004/051. http://ssrn.com/abstract $=622743$

Kim, T.S., and E. Omberg, (1996), Dynamic nonmyopic portfolio behavior, Review of Financial Studies, 9, 141-61.

Kimball, M.S., (1990), Precautionary savings in the small and in the large, Econometrica, 58, 53-73.

Kogan, L., and R. Uppal, (2000), Risk aversion and optimal portfolio policies in partial and general equilibrium economies, http://finance.commerce.ubc.ca/ ^uppal/papers.html.

Leland, H.E., (1980), Who Should Buy Portfolio Insurance?, Journal of Finance, 35, 581-596.

Merton, R.C., (1969), Lifetime portfolio selection under uncertainty: The continuous time case, Review of Economics and Statistics, 51, 247-257.

Merton, R.C., (1971), Optimum consumption and portfolio rules in a continuous-time model, Journal of Economic Theory, 3, $373-413$.

Mossin, J., (1968), Optimal multiperiod portfolio policies, Journal of Business, 215-229.

Poterba, J., and L.H. Summers, (1988), Mean reversion in stock returns: Evidence and implications, Journal of Financial Economics, 22, 27-60. 
Rothschild, M. and J. Stiglitz, (1971), Increasing risk: II Its economic consequences, Journal of Economic Theory 3, 66-84.

Sandmo, A., (1970), The effect of uncertainty on saving decisions, Review of Economic Studies, 37, 353-360.

Vasicek, 0., (1977), An equilibrium characterization of the term structure, Journal of Financial Economics, 5, 177-188.

Wilson, R. (1968). "The theory of syndicates", Econometrica 36, 113-132. 


\section{Appendix : Proof of Proposition 1}

Proof: We prove that an increase in the risk free rate raises the marginal value of wealth when the index of relative risk aversion is uniformly less than unity. A symmetric proof can be written for the case with $R \geq 1$. Observe that $R(c) \leq 1$ if and only if $T(c) \geq c$. Thus, our assumption in this proof is that $T(c) \geq c$ for all $c \geq 0$. We need to prove that

$$
H=1-\frac{[\widehat{E} \widetilde{x}]^{2}}{\widehat{E} \widetilde{x}^{2}}-\frac{\widehat{E} T(y+\widetilde{x})}{y} \leq 0
$$

for all $(y, \widetilde{x})$ such that $\widehat{E} \widetilde{x} T(y+\widetilde{x})=0$. Our strategy is to search for the function $T$ that is the best candidate to violate condition $H \leq 0$. In order to escape technical difficulties, we suppose that $\widetilde{x}$ has a bounded support in $[a, b]$. Of course, we must have $a<0<b$, and $y+a>0$. Let $G$ denote the risk-neutral cumulative distribution function of $\widetilde{x}$. The best candidate is obtained by solving the following program:

$$
\begin{aligned}
& \min _{T(.)} \int_{a}^{b} T(y+x) d G(x) \\
& \text { s.t. } \quad 0=\int_{a}^{b} x T(y+x) d G(x) \\
& T(y+x) \geq y+x \quad \forall x \in[a, b] .
\end{aligned}
$$

Using the standard technique of the calculus of variation, the necessary and sufficient conditions to this problem are written as, for all $x$,

$$
1+\lambda x\left\{\begin{array}{lll}
=0 & \text { if } & T(y+x)>y+x \\
\geq 0 & \text { if } & T(y+x)=y+x
\end{array}\right.
$$

Two cases must be considered depending upon the sign of $\widehat{E} \widetilde{x}(y+\widetilde{x})$.

- Consider first the case where $\widehat{E} \widetilde{x}(y+\widetilde{x}) \geq 0$. In that case, conditions (21) are satisfied with $\lambda$ equaling $-1 / a$, and

$$
T(y+x)=y+x-\frac{\widehat{E} \widetilde{x}(y+\widetilde{x})}{a} \delta_{a}(x)
$$


for all $x \in[a, b]$, where $\delta_{a}($.$) is the Dirac function taking value zero$ everywhere except when $x=a$, with $\int \delta_{a}(x) d G(x)=1$. It implies that the smallest value of $\widehat{E} T$ equals

$$
\widehat{E} T(y+\widetilde{x})=\frac{\widehat{E}(a-\widetilde{x})(y+\widetilde{x})}{a} .
$$

From this, we deduce that the largest possible $H$ under this risk-neutral distribution of $\widetilde{x}$ is equal to

$$
H=\frac{\widehat{E}[\widetilde{x}(y+\widetilde{x})] \widehat{E}[\widetilde{x}(\widetilde{x}-a)]}{a y \widehat{E} \widetilde{x}^{2}} .
$$

We want to prove that this $H$ is negative. Suppose by contradiction that it is strictly positive, i.e., that

$$
\widehat{E} \widetilde{x}(\widetilde{x}-a)<0 \text { and } \widehat{E} \widetilde{x}(y+\widetilde{x})>0
$$

or, equivalently, that

$$
-y \widehat{E} \widetilde{x}<\widehat{E} \widetilde{x}^{2}<a \widehat{E} \widetilde{x} .
$$

Observe first that $-y \widehat{E} \widetilde{x}<a \widehat{E} \widetilde{x}$ implies that $\widehat{E} \widetilde{x}$ must be strictly positive, since $-y<a$. Observe also that

$$
\widehat{E} \widetilde{x}^{2} \geq(\widehat{E} \widetilde{x})^{2} \geq a \widehat{E} \widetilde{x} .
$$

The second inequality comes from combining $\widehat{E} \widetilde{x}>0$ and $\widehat{E} \widetilde{x} \geq a$. Inequality (22) implies that $\widehat{E} \widetilde{x}(\widetilde{x}-a)$ is positive, a contradiction. Thus, the largest possible $H$ is negative and the result is proved when $\widehat{E} \widetilde{x}(y+$ $\widetilde{x}) \geq 0$.

- Let us now consider the second possible case where $\widehat{E} \widetilde{x}(y+\widetilde{x})$ is negative. In that case, the solution to $(21)$ is with $\lambda=-1 / b$, and

$$
T(y+x)=y+x-\frac{\widehat{E} \widetilde{x}(y+\widetilde{x})}{b} \delta_{b}(x)
$$

for all $x \in[a, b]$, where $\delta_{b}($.$) is the Dirac function taking value zero$ everywhere except when $x=b$, with $\int \delta_{b}(x) d G(x)=1$. After some tedious manipulations, we obtain for this optimal $T$ function that

$$
\widehat{E} T(y+\widetilde{x})=\frac{\widehat{E}(b-\widetilde{x})(y+\widetilde{x})}{b} .
$$


It implies that the largest possible $H$ under this risk-neutral distribution of $\widetilde{x}$ is equal to

$$
H=\frac{\widehat{E}[\widetilde{x}(y+\widetilde{x})] \widehat{E}[\widetilde{x}(\widetilde{x}-b)]}{b y \widehat{E} \widetilde{x}^{2}} .
$$

We want to prove that this $H$ is negative. Suppose by contradiction that it is strictly positive, i.e., that

$$
\widehat{E} \widetilde{x}(\widetilde{x}-b)<0 \text { and } \widehat{E} \widetilde{x}(y+\widetilde{x})<0
$$

or, equivalently, that

$$
\left\{\begin{array}{c}
\widehat{E} \widetilde{x}^{2}<b \widehat{E} \widetilde{x} \\
\widehat{E} \widetilde{x}^{2}<-y \widehat{E} \widetilde{x}
\end{array}\right.
$$

Observe first that $\widehat{E} \widetilde{x}^{2}<-y \widehat{E} \widetilde{x}$ is possible only if $\widehat{E} \widetilde{x}$ is strictly negative. Observe also that

$$
\widehat{E} \widetilde{x}^{2} \geq(\widehat{E} \widetilde{x})^{2} \geq b \widehat{E} \widetilde{x}
$$

The second inequality comes from the fact that $\widehat{E} \widetilde{x}<0$ and $\widehat{E} \widetilde{x} \leq b$. Inequality (23) implies that $\widehat{E} \widetilde{x}(\widetilde{x}-b)$ is positive, a contradiction. This concludes the proof of Proposition 1. 